\title{
Expression and Localization of Prestin and the Sugar Transporter GLUT-5 during Development of Electromotility in Cochlear Outer Hair Cells
}

\author{
Inna A. Belyantseva, ${ }^{1}$ Henry J. Adler, ${ }^{1}$ Rui Curi, ${ }^{2}$ Gregory I. Frolenkov, ${ }^{1}$ and Bechara Kachar ${ }^{1}$ \\ ${ }^{1}$ Section on Structural Cell Biology, National Institute on Deafness and Other Communication Disorders, National \\ Institutes of Health, Bethesda, Maryland 20892, and 2Department of Physiology and Biophysics, Institute of Biomedical \\ Sciences, University of Sao Paulo, Brazil
}

Electromotility, i.e., the ability of cochlear outer hair cells $(\mathrm{OHCs})$ to contract and elongate at acoustic frequencies, is presumed to depend on the voltage-driven conformational changes of "motor" proteins present in the OHC lateral plasma membrane. Recently, two membrane proteins have been proposed as candidates for the $\mathrm{OHC}$ motor. A sugar transporter, GLUT-5, was proposed based on its localization in the OHCs and on the observation that sugar transport alters the voltage sensitivity of the $\mathrm{OHC}$ motor mechanism. Another candidate, "prestin," was identified from a subtracted OHC cDNA library and shown to impart voltage-driven shape changes to transfected cultured cells. We used antibodies specific for these two proteins to show that they are highly expressed in the lateral membrane of OHCs. We also compared the postnatal expression patterns of these proteins with the development of elec- tromotility in OHCs of the apical turn of the rat organ of Corti. The patch-clamp recording of transient charge movement associated with electromotility indicates that half of the maximal expression of the motor protein occurs at postnatal day 9 . Prestin incorporation in the plasma membrane begins from postnatal day 0 and increases progressively in a time course coinciding with that of electromotility. GLUT-5 is not incorporated into the lateral plasma membrane of apical $\mathrm{OHCs}$ until postnatal day 15. Our results suggest that, although GLUT-5 may be involved in the control of electromotility, prestin is likely to be a fundamental component of the $\mathrm{OHC}$ membrane motor mechanism.

Key words: mechanosensory transduction; unconventional cell motility; motor protein; organ of Corti; postnatal development; voltage-dependent capacitance
The hearing sensitivity in mammals depends on the function of special mechanosensory cells, outer hair cells (OHCs), which not only sense sound-induced vibrations in the organ of Corti but also amplify them. This amplification is generally believed to result from the ability of OHCs to change length when their intracellular potential is changed. This unique type of cell motility, called electromotility, operates at acoustic frequencies (Dallos and Evans, 1995) and does not require ATP hydrolysis (Kachar et al., 1986).

The molecular mechanism for electromotility is based on voltage-dependent conformational changes of "motor" proteins densely packed in the lateral plasma membrane (Kalinec et al., 1992; Frolenkov et al., 1998). These conformational changes are experimentally detected as voltage-dependent changes in the membrane capacitance (Santos-Sacchi, 1991; Ashmore, 1992) and changes of the surface area of the membrane (Kalinec et al., 1992). The surface area changes are presumed to be translated into changes in cell length by the anisotropic cytoskeleton of the OHC (Holley et al., 1992).

The fast operation and insensitivity to ion channel blockers

Received Aug. 11, 2000; revised Sept. 20, 2000; accepted Sept. 21, 2000.

This work was supported by National Institute on Deafness and Other Communication Disorders Intramural Research Project Z01 DC 0002-11 and in part by Fundação de Amparo à Pesquisa do Estado de São Paulo Grant 98/11714-2 (R.C.). We thank Drs. Peter Dallos, Mark Schneider, and Ron Petralia for their comments on this manuscript.

Correspondence should be addressed to Dr. Bechara Kachar, Section on Structural Cell Biology, National Institute on Deafness and Other Communication Disorders, National Institutes of Health, Building 36, Room 5D15, Bethesda, MD 20892-4163. E-mail: kacharb@nidcd.nih.gov.

Copyright (๑) 2000 Society for Neuroscience $0270-6474 / 00 / 200001-05 \$ 15.00 / 0$
(Frolenkov et al., 1998) indicates that the motor protein is more likely to be a transporter rather than a modified ion channel (Geleoc et al., 1999). Recently, two molecules have been proposed as candidates for the OHC motor: the facilitative glucose transporter GLUT-5 (Geleoc et al., 1999), and a new protein, prestin, containing a sulfate-transporting motif (Zheng et al., 2000). GLUT-5 was proposed as an OHC motor based on its localization in the OHC lateral plasma membrane (Nakazawa et al., 1995) and on the fact that sugar transport alters voltage sensitivity of the OHC motor mechanism (Geleoc et al., 1999). Prestin was identified from a pool of proteins highly expressed in OHCs and not expressed in nonmotile inner hair cells (IHCs) using a cDNA library subtraction procedure (Zheng et al., 2000). Cultured kidney cells transfected with prestin cDNA showed voltage-dependent capacitance and electrically evoked changes of cell shape (Zheng et al., 2000). The localization of prestin in OHCs has not been investigated.

This article is published in The Journal of Neuroscience, Rapid Communications Section, which publishes brief, peerreviewed papers online, not in print. Rapid Communications are posted online approximately one month earlier than they would appear if printed. They are listed in the Table of Contents of the next open issue of JNeurosci. Cite this article as: JNeurosci, 2000, 20:RC116 (1-5). The publication date is the date of posting online at www.jneurosci.org.

http://www.jneurosci.org/cgi/content/full/4792 
In rat and gerbil, structural and functional maturation of the organ of Corti (Pujol et al., 1980; Rubel, 1984) and appearance of OHC electromotility (He et al., 1994) occur within the first 2 weeks after birth. A candidate protein for $\mathrm{OHC}$ motor should be expressed before or at the time of maturation of OHC electromotility. We have now used antibodies specific for GLUT-5 and prestin to show by immunofluorescence that these proteins are highly expressed in the lateral plasma membrane of OHCs in the region in which electromotility is generated. We also compared the pattern of expression of each protein to the time course of development of electromotility in the OHCs from the apical turn of the cochlea.

\section{MATERIALS AND METHODS}

Immunocytochemistry. Sprague Dawley rats (Taconic, Germantown, NY), either adults $(120-150 \mathrm{gm})$ or pups ranging from postnatal day 0 (PD0) to PD22, were suffocated with $\mathrm{CO}_{2}$ and decapitated according to National Institutes of Health Guidelines for Animal Use. The bullae were removed, and the cochleae were perfused through the round window with $4 \%$ paraformaldehyde in PBS and incubated in this fixative for $1 \mathrm{hr}$ at room temperature $\left(22-24^{\circ} \mathrm{C}\right)$. The organ of Corti was dissected from the cochlear spiral in PBS using a fine needle. Samples were permeabilized in $0.5 \%$ Triton X-100 for $30 \mathrm{~min}$ and then washed in PBS. Nonspecific binding sites were blocked using 5\% normal goat serum (Life Technologies, Gaithersburg, MD) and $2 \%$ bovine serum albumin (ICN, Aurora, $\mathrm{OH}$ ) in PBS for $2 \mathrm{hr}$. Samples were incubated for $2 \mathrm{hr}$ in the primary antibodies at a concentration of $\sim 5 \mu \mathrm{g} / \mathrm{ml}$ in blocking solution. After several rinses in PBS, samples were incubated in a 1:200 dilution of the fluorescein-conjugated anti-rabbit IgG secondary antibody (Amersham Pharmacia Biotech, Arlington Heights, IL) for $40 \mathrm{~min}$. Samples were mounted using ProLong Antifade Kit (Molecular Probes, Eugene, OR) and viewed with a Zeiss (Oberkochen, Germany) Axiophot and a 510 Zeiss Confocal microscopes.

Antibodies. Commercially available antibodies against GLUT-5 (cata$\log$ \#4670-1756, Biogenesis, Brentwood, NH; and catalog \#AB1048, Chemicon, Temecula, CA) were used in this study. To produce antibodies against prestin, rabbits were immunized (Covance, Denver, PA) with synthetic peptides (Princeton Biomolecules, Langhome, PA) corresponding to the portions of the gerbil prestin (Zheng et al., 2000) (GenBank accession number AF230376). Two rabbits were immunized with a peptide from the $\mathrm{C}$ terminus domain (amino acids 725-744, SAPPPQDDMEPNATPTTPEA), and two rabbits were immunized with a peptide from the $\mathrm{N}$ terminus domain (amino acids 19-34, KYHVERPIFSHPVLQE). All four rabbits provided high antibody titer. Because the amino acid homology between human and gerbil prestin, deduced from the genomic sequence of the first six exons, is 98\% (Zheng et al., 2000), we expected that our polyclonal antibodies would cross-react with rat prestin.

Immunofluorescence quantification. For the quantification of developmental changes of GLUT-5 and prestin expression in the OHC plasma membrane, we calculated relative values of immunofluorescence signals from conventional fluorescence micrographs taken at the same exposure times from simultaneously processed specimens. The $35 \mathrm{~mm}$ film was digitized. Equally sized images containing $14 \mathrm{OHCs}$ were analyzed using MetaMorph (Universal Imaging, West Chester, PA) image processing software. To equalize the background intensities (which varied by $\sim 30 \%$ ) to a new baseline level common for all images, we used the MetaMorph "shadow correction" function (Russ, 1999). The histograms of pixel intensities were plotted for each image, and the gaussian-shaped intensity distribution of the background was subtracted from each histogram. Finally, we calculated the integral brightness (integral of the number of pixels multiplied by the respective intensity values) of the subtracted histogram. This value represents the total intensity of fluorescence from the OHC plasma membrane when no cytoplasmic structures were labeled. When there was also labeling in the cytoplasm (in the case of GLUT-5), this labeling was excluded from the calculation by masking circular areas $(6 \mu \mathrm{m}$ in diameter) located in the center of each OHC.

Patch-clamp recording. OHCs from the apical turn of the organ of Corti, which are easier to isolate and to study electromotile responses, were isolated in a modified Leibowitz cell culture medium (L-15; osmolarity, $325 \pm 2$ mOsm; pH $7.4 \pm 0.1$ ) as described previously (Frolenkov et al., 2000). Isolated OHCs were placed in a $100 \mu$ l laminar flow bath with a $5 \mathrm{ml} / \mathrm{hr}$ exchange rate and maintained at room temperature throughout the experiments. The viability of selected OHCs visualized on the microscope slide was determined based on the following: uniform cylindrical shape, basal location of the nucleus, and intact stereocilia.

Patch-clamp recordings were performed using an Axopatch 1D amplifier (Axon Instruments, Foster City, CA). Pipettes for whole-cell recordings were formed on a programmable puller (P87; Sutter Instruments, Novato, CA) from $1.0 \mathrm{~mm}$ outer diameter borosilicate glass (\#30-30-0; Frederick Haer \& Co. Inc., Bowdoinham, ME) and filled with an intracellular solution containing (in mM): $\mathrm{CsCl} 140, \mathrm{MgCl}_{2}$ 2.0, EGTA 5.0, and HEPES 5.0, adjusted to $\mathrm{pH} 7.2$ with $\mathrm{CsOH}$ and brought to 325 mOsm with D-glucose. Current and voltage were sampled at $100 \mathrm{kHz}$ using an interface (Digidata 1200A; Axon Instruments) controlled by pClamp 7.0 software (Axon Instruments). Potentials were corrected off-line for the error attributable to access resistance.

Membrane capacitance measurement. Plasma membrane capacitance, $C_{m}$, was measured using the "membrane test" feature of the pClamp 7.0 acquisition software (Frolenkov et al., 2000). The voltage-independent fraction of the cell capacitance scales linearly with the overall surface area of the cell. However, the nonlinear voltage-dependent fraction of the cell capacitance is proportional to the area of the lateral membrane containing the putative motor elements (Huang and Santos-Sacchi, 1993). Therefore, to compare the data obtained from different cells, the nonlinear voltage-dependent capacitance was divided by the area of the lateral plasma membrane to yield the specific nonlinear voltagedependent capacitance:

$$
C_{n l i n}(V)=\left(C_{m}(V)-C_{0}\right) /[\pi D(L-D / 2)],
$$

(in microfarads per square centimeters), where $L$ is the cell length, $D$ is the diameter, and $C_{0}$ is the voltage-independent fraction of the cell capacitance. To estimate the density of charge movement (in elementary

charges per square micrometer), we computed $\int_{-\infty}^{+\infty} C_{n l i n}(V) d V$ by numerical integration. This density is proportional to the density of motor proteins in the OHC lateral plasma membrane (Santos-Sacchi, 1991; Ashmore, 1992).

Motility measurements. Motility measurements were performed as described previously (Frolenkov et al., 1997). Briefly, OHC images were analyzed off-line with the image-processing system Image 1 (Universal Imaging). For movement quantification, a measuring rectangle ranging in length from 5 to $20 \mu \mathrm{m}$ and composed of 3-15 rows of pixels was positioned across the moving edge of the cell. The average intensity profile across the edge of the cell was calculated, and the number of points in profile was increased 10 times by cubic spline interpolation. Movement of the cell edge was calculated from the frame-by-frame shift (computed by a least-square procedure) in the interpolated intensity profiles. The sensitivity of the measurement was $\sim 0.02 \mu \mathrm{m}$, as determined previously (Frolenkov et al., 1997).

\section{RESULTS}

\section{Immunolocalization of GLUT-5 and prestin in OHCs of adult rat}

In agreement with previously published data (Nakazawa et al., 1995), the two anti-GLUT-5 antibodies labeled the lateral plasma membrane of the OHCs. The labeling was observed as an annular fluorescence pattern in optical cross-sections of the whole-mount preparation of the organ of Corti (Fig. 1A). The labeling extended from the top of the OHCs, just below the cuticular plate at the stereociliary pole, to the level of the nucleus at the synaptic pole. Some punctate labeling was observed in the cytoplasm of OHCs from the apical turn of the organ of Corti (data not shown). A comparatively weak labeling could also be observed near the apical region of IHCs and phalangeal processes of the inner pillar cells (Fig. 1A). Preincubation of the antibodies with an excess of the immunizing peptide ( $1 \mathrm{hr}$ at room temperature) eliminated the labeling (Fig. 1B), confirming its specificity. Also, incubation with the secondary antibody alone did not produce any labeling (data not shown).

The immunofluorescence labeling in the adult organ of Corti 

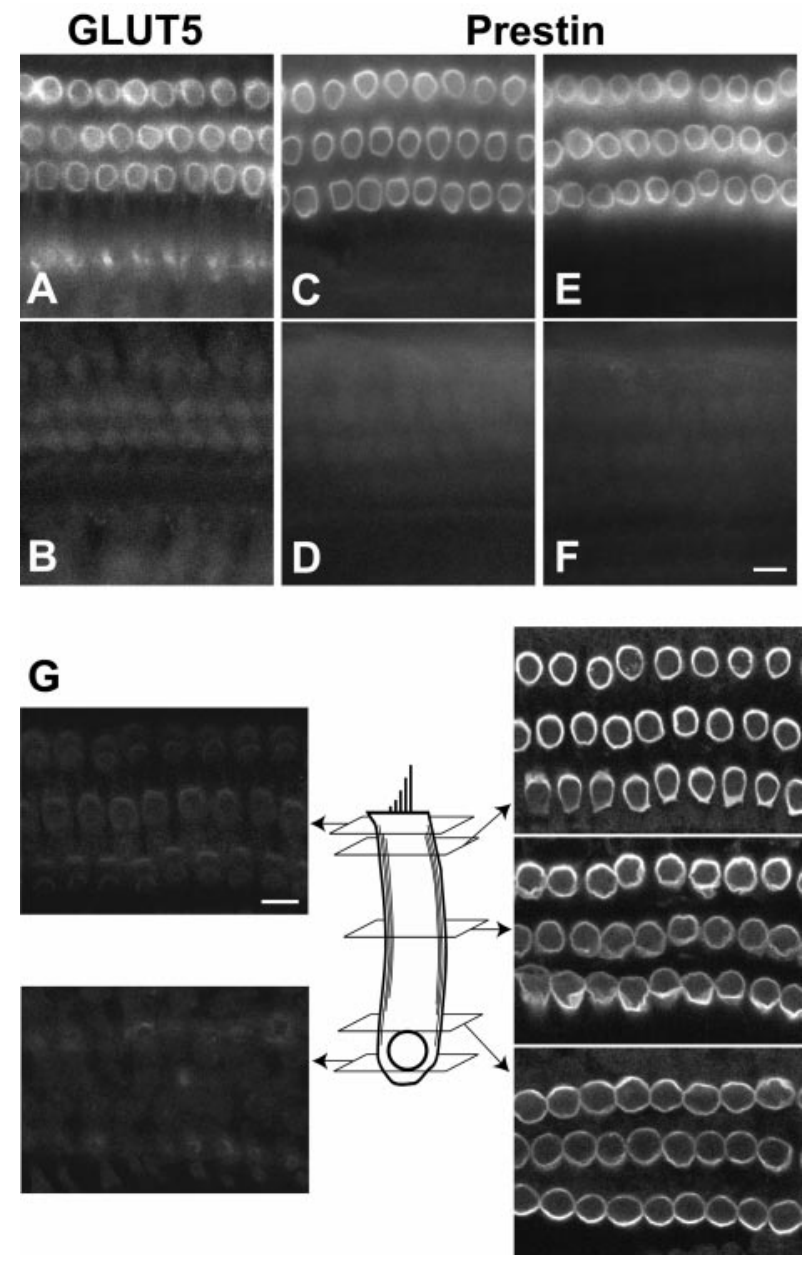

Figure 1. Immunolocalization of GLUT-5 and prestin in the organ of Corti of adult rat. $A, C, E$, Conventional fluorescent image of wholemount preparations labeled with the anti-GLUT-5 (Chemicon) $(A)$, antiprestin (C terminus) $(C)$, and anti-prestin ( $\mathrm{N}$ terminus) $(E)$ antibodies. All antibodies distinctly labeled the lateral wall of the OHCs, producing annular fluorescence patterns. $B, D, F$, Immunolabeling after preadsorption of the primary antibodies with the corresponding immunizing peptides. $G$, Serial confocal cross-sections $(0.8 \mu \mathrm{m}$ thickness $)$ of samples labeled with the anti-prestin ( $\mathrm{C}$ terminus) antibody taken at different levels along the $\mathrm{OHC}$ body as indicated in the diagram. Scale bars, $10 \mu \mathrm{m}$.

with all four rabbit anti-prestin antisera that we produced was specific to the OHCs (not present in any other cells) and concentrated along the lateral membrane. Figure $1, C$ and $E$, shows the pattern of labeling obtained with antisera to the $\mathrm{C}$ - and $\mathrm{N}$-termini prestin peptides, respectively. No labeling of the OHCs was observed when the antibodies were preincubated with the respective immunizing peptides (Fig. $1 D, F$ ). Again, the bright labeling of the lateral membrane of the OHCs extended uniformly from the region just below the level of the cuticular plate to the level of the nucleus (Fig. $1 G$ ), as demonstrated with the series of confocal images. No labeling was detected in the stereocilia in the junctional region around the cuticular plate, in the subnuclear region, or in the cytoplasm of the OHCs.

\section{Postnatal development of GLUT-5 immunoreactivity}

We investigated the developmental changes of GLUT-5 immunoreactivity in the rat organ of Corti at different ages between PD0 and PD22. GLUT-5 immunoreactivity was first found as a punctate labeling in the cytoplasm of OHCs and IHCs as early as PD3 (data not shown). No labeling in the lateral wall of OHCs

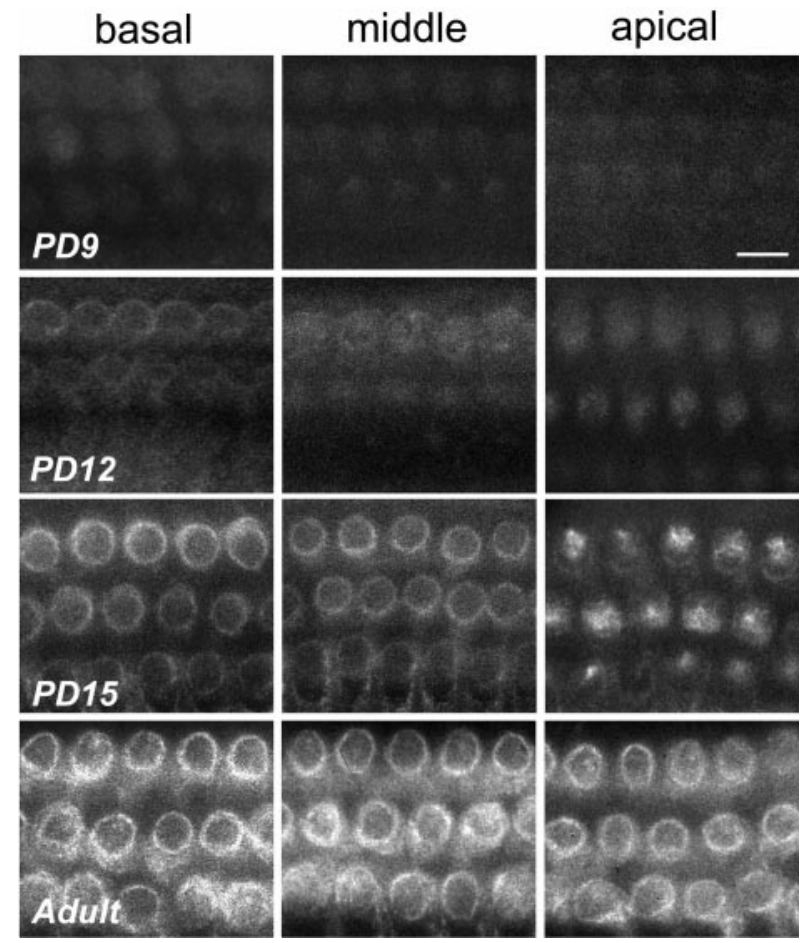

Figure 2. Postnatal development of GLUT-5 immunoreactivity in the rat organ of Corti. Organs of Corti from 9-d-old (PD9), 12-d-old (PD12), 15-d-old (PD15), and adult rats were separated into three segments (basal, middle, and apical) and processed simultaneously. Each panel shows a conventional fluorescence microscopy image of whole-mount preparations taken at a plane of focus approximately at the middle of the OHCs. Scale bar, $10 \mu \mathrm{m}$.

was observed until PD12 (Fig. 2). At PD12, labeling of the lateral wall was detected in $~ 95 \%$ of OHCs from the basal turn and in $50 \%$ of OHCs from the middle turn but was not present in apical OHCs. In OHCs from the apical turn, labeling started to appear in the lateral wall only at PD15 and reached the maximal intensity levels at PD19 (Fig. 2). After the incorporation of GLUT-5 into the plasma membrane, immunoreactivity in the cytoplasm gradually decreased and became not detectable, except for some punctate labeling in the most apical OHCs (data not shown). GLUT-5 immunoreactivity was not detected in the lateral plasma membrane of IHCs, but it was present in the cytoplasm of IHCs between PD3 and PD10. In adult rats, only the IHCs from the apical turn showed this cytoplasmic labeling.

\section{Postnatal development of prestin immunoreactivity in the organ of Corti}

In contrast to GLUT-5, prestin was detected in the lateral wall of OHCs as early as PD0 (Fig. 3). The most prominent increase in the intensity of $\mathrm{OHC}$ lateral membrane labeling occurred between PD6 and PD9. The intensity of labeling reached adult levels at PD9 in the basal turn of the cochlea, at PD10-PD11 in the middle turn, and at PD12 in the apical turn (Fig. 3). Prestin immunoreactivity was never observed in other cells of the organ of Corti.

\section{Postnatal development of OHC electromotility in the apical turn of the cochlea}

The translocation of electrical charges across the plasma membrane accompanies OHC electromotility and imparts a bellshaped dependence of membrane capacitance on transmembrane potential (Santos-Sacchi, 1991). Using whole-cell patch clamp, we detected electromotility and voltage-dependent capacitance in iso- 


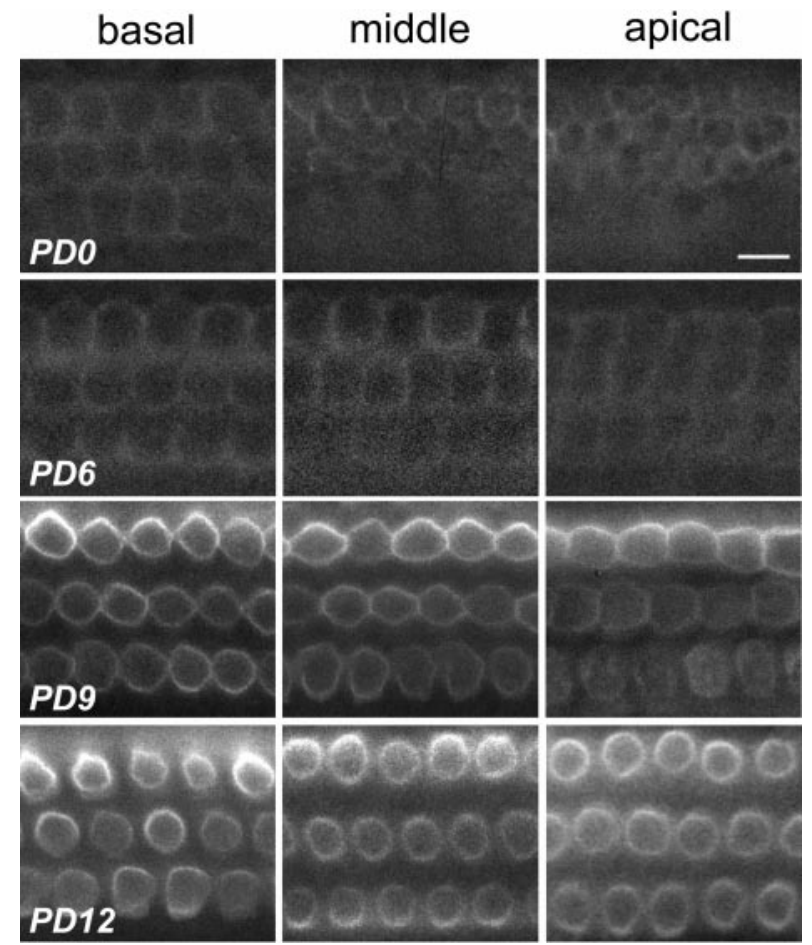

Figure 3. Postnatal development of prestin immunoreactivity in the rat organ of Corti. Organs of Corti from neonatal (PDO), 6-d-old (PD6), 9-d-old (PD9), and 12-d-old (PD12) rats were separated into three segments (basal, middle, and apical) and processed simultaneously using the anti-prestin (C terminus) antibody. Each panel shows a conventional fluorescence microscopy image of whole-mount preparations taken at a plane of focus approximately at the middle of the OHCs. Scale bar, $10 \mu \mathrm{m}$.

lated OHCs as early as P5 (Fig. 4A). Electromotile responses and voltage-dependent capacitance progressively increased with the steepest increase between PD8 and PD12. At PD12, OHCs showed robust electromotility and the characteristic bell-shaped voltage dependence of the capacitance. At this age, the density of the motility-associated charge movement reached the value of $5450 \pm$ $340 \mathrm{e}^{-} / \mu \mathrm{m}^{2}(n=9)$, close to the value of $7200 \pm 720 \mathrm{e}^{-} / \mu \mathrm{m}^{2}(n=$ 8 ) measured in the adult OHCs. Half of the maximal density of motor proteins was reached at approximately PD9 (Fig. 4B).

\section{Development of electromotility versus prestin and GLUT-5 immunoreactivity}

It is apparent from immunofluorescence data that prestin expression in the OHC lateral plasma membrane developed concurrently with OHC electromotility, whereas GLUT-5 appeared in OHC plasma membrane a few days later. For comparison, we estimated values of relative immunofluorescence intensity in the apical turn of the cochlea and plotted them in a time course graph with the values of density of motor proteins estimated from the measurements of capacitance (Fig. 4B). This graph shows that the developmental increase of prestin immunoreactivity clearly coincided with the increase of the density of OHC motor proteins. Although the GLUT-5 expression in the plasma membrane of OHCs shows a similar increase, its time course was delayed by $\sim 6$ d compared with that of prestin and electromotility development (Fig. $4 B$ ). In an additional control experiment, we used a 12-d-old rat in which the apical turn of one cochlea was processed for GLUT-5 immunofluorescence and the apical turn of the contralateral cochlea for electromotility studies. No immunofluorescence labeling was observed in the lateral plasma membrane,
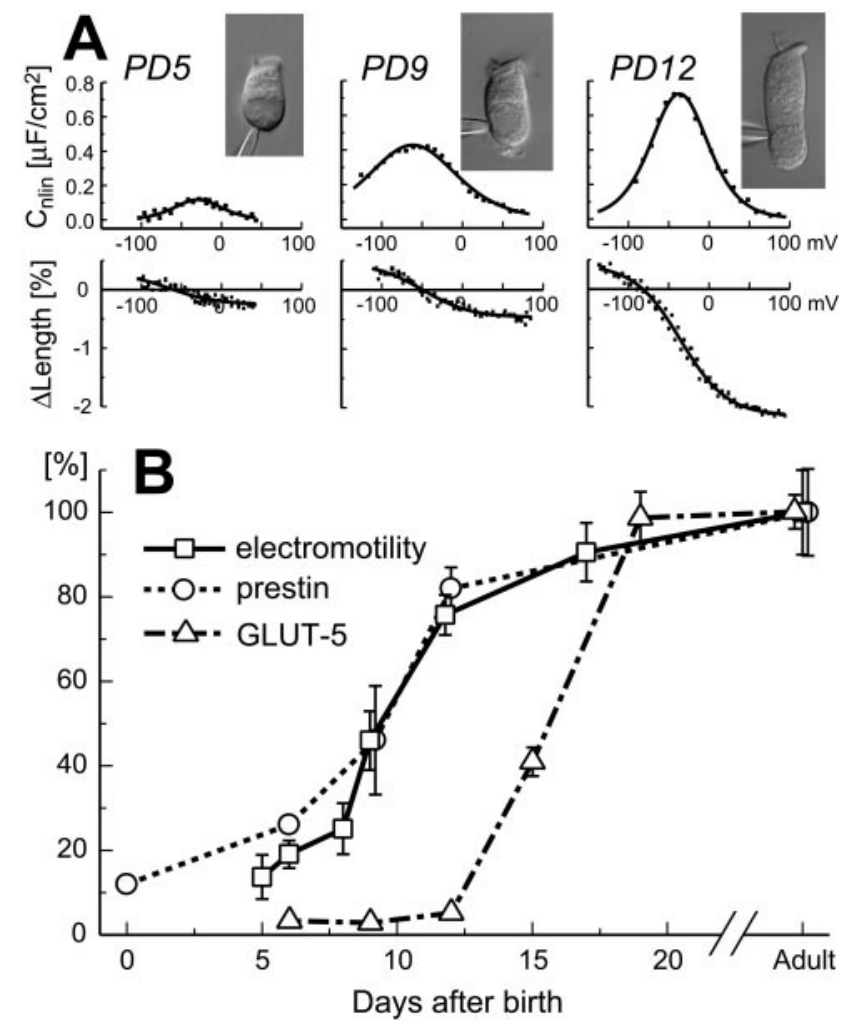

Figure 4. Postnatal increase of electromotility and motility-associated charge movement compared with that of prestin and GLUT-5 immunoreactivity in the OHCs of the apical turn of the organ of Corti. $A$, Plots of the specific nonlinear capacitance $\left(C_{\text {nlin }}\right.$, top row $)$ and length changes $(\Delta$ Length, bottom row) versus transmembrane voltage for three OHCs (video images shown as insets) at postnatal days 5 (PD5), 9 (PD9), and 12 (PD12). Capacitance data are fitted with the derivative of a Boltzmann function. Length changes are expressed in percentage of the cell length at a holding potential of $-60 \mathrm{mV}$ and fitted with a Boltzmann function. $B$, Density of the electromotility-associated charge movement (squares) and intensity of immunolabeling of OHC lateral wall with anti-prestin (circles) and antiGLUT-5 (triangles) antibodies versus days after birth (mean $\pm \mathrm{SE}$ ). Values obtained from several OHCs (for capacitance, $n=3-11$; for immunofluorescence, $n=14$ ) were averaged and then normalized to the corresponding average value observed in adult rats. SEs were scaled accordingly.

whereas the $\mathrm{OHCs}$ from the contralateral cochlea showed robust electromotility and significant voltage-dependent capacitance $\left(0.5-0.9 \mu \mathrm{F} / \mathrm{cm}^{2} ; n=6\right)$.

\section{DISCUSSION}

\section{GLUT-5 is unlikely to be the motor protein of OHC}

We confirmed previously reported data (Nakazawa et al., 1995) that GLUT-5 is highly expressed in the lateral wall of OHCs, at the expected location for the OHC motor proteins (Dallos et al., 1991; Kalinec et al., 1992). However, GLUT-5 was not detected in the lateral wall of apical OHCs until PD15. At PD15, OHCs exhibited vigorous electromotility, and the density of $\mathrm{OHC}$ motor proteins in the lateral plasma membrane was already close to the maximal density observed in adult animals (Fig. 4) (Oliver and Fakler, 1999). Thus, we concluded that the development of OHC electromotility precedes the incorporation of GLUT-5 in the OHC plasma membrane, and therefore this protein is unlikely to be the motor element for electromotility. Furthermore, electromotility is known to be present only in OHCs (Kachar et al., 1986), but we and others have observed some GLUT-5 expression in IHCs (Geleoc et al., 1999) and supporting cells (Nakazawa et al., 1995).

Although GLUT-5 is not the motor protein, it may be involved 
in the control of electromotility. GLUT-5 appears in the plasma membrane of OHCs immediately after the onset of hearing function at PD12 (Crowley and Hepp-Reymond, 1966). This is the time when auditory thresholds rapidly decrease toward values typical for adult animals (Pujol et al., 1980; Uziel et al., 1981). It is also the time of appearance of abundant deposits of glycogen particles in the OHC cytoplasm (Rueda et al., 1990). GLUT-5 may contribute to the transport of sugars and maintenance of glycogen stores. Energy-consuming $\mathrm{Ca}^{2+}$-dependent mechanisms regulate shape, stiffness, and motor output of $\mathrm{OHCs}(\mathrm{Du}-$ lon et al., 1990; Dallos et al., 1997; Frolenkov et al., 2000). A system of endoplasmic reticulum membranes, the subsurface cisternae, and a high density of mitochondria are found near the lateral plasma membrane in which electromotility is generated. The demonstration of abundant $\mathrm{Ca}^{2+}$ pumps in the subsurface cisternae confirms the role of this organelle as an intracellular reservoir for $\mathrm{Ca}^{2+}$ (Schulte, 1993). Notably, a rapid increase in the expression of the $\mathrm{Ca}^{2+}$-ATPase pumps in the subsurface cisternae (Schulte, 1993) occurs at approximately the same postnatal period when GLUT-5 appears in the plasma membrane. $\mathrm{Ca}^{2+}$ is a well known activator of glycogen breakdown and oxidative metabolism in mitochondria (Newsholme and Leech, 1983). All of the above facts indicate that a high-energy consumption system develops in this region of the cell at the time of appearance of GLUT-5 in the plasma membrane. This system may be involved in buffering $\mathrm{Ca}^{2+}$ and regulating $\mathrm{OHC}$ electromotility (Frolenkov et al., 2000).

GLUT-5 is probably incorporated into the lateral plasma membrane among the motor proteins, so that both proteins may contribute to the extremely high density of intramembrane particles observed in OHCs (Gulley and Reese, 1977; Forge, 1991; Kalinec et al., 1992). Such an environment may be conducive to lateral proteinprotein interactions. These potential interactions may explain voltage dependence of sugar transport in $\mathrm{OHCs}$, as well as its influence on the voltage sensitivity of OHC motors, which was the basis for proposing GLUT-5 as the OHC motor (Geleoc et al., 1999).

\section{Evidence that prestin is the $\mathrm{OHC}$ motor}

Prestin was identified from an OHC cDNA library, after suppression subtraction hybridization, as a protein highly expressed in electromotile OHCs but not in nonmotile IHCs (Zheng et al., 2000). Cultured kidney cells transfected with prestin exhibit voltage-dependent capacitance and electrically evoked shape changes (Zheng et al., 2000), suggesting that prestin undergoes voltage-driven conformational changes associated with alterations of the membrane area similar to what occurs in OHC electromotility. Here we used two different anti-prestin antibodies to provide the first direct evidence for the localization of prestin in the lateral plasma membrane of OHCs in the same region in which electromotility occurs. Prestin is exclusively expressed in the lateral plasma membrane of the OHCs, as would be expected for the protein responsible for electromotility (Kalinec et al., 1992). No other regions of the $\mathrm{OHC}$ plasma membrane or any other cells in the organ of Corti were labeled with the anti-prestin antibodies. Our data showing that the time course of postnatal development of prestin immunoreactivity coincides with that of electromotility further support the hypothesis that prestin is likely to be an OHC motor protein.

The OHC motor has been proposed to be a modified anion exchanger (Kalinec and Kachar, 1993). Prestin contains a sulfate transport motif and has $\sim 40 \%$ homology to anion transport proteins (Zheng et al., 2000). Whether prestin has any transport function is unknown. It has still to be investigated how prestin as a potential OHC motor may sense transmembrane voltage and produce substantial changes of the membrane area. The antibodies that we generated for the specific sequences at the $\mathrm{C}$ and $\mathrm{N}$ termini of prestin should be useful for testing functional domains and topology models of this novel membrane molecule and as specific cell markers for OHCs in developmental studies of the organ of Corti.

\section{REFERENCES}

Ashmore JF (1992) Mammalian hearing and the cellular mechanisms of the cochlear amplifier. Soc Gen Physiol Ser 47:395-412.

Crowley DE, Hepp-Reymond MC (1966) Development of cochlear function in the ear of the infant rat. J Comp Physiol Psychol 62:427-432.

Dallos P, Evans BN (1995) High-frequency motility of outer hair cells and the cochlear amplifier. Science 267:2006-2009.

Dallos P, Evans BN, Hallworth R (1991) Nature of the motor element in electrokinetic shape changes of cochlear outer hair cells. Nature 350:155-157.

Dallos P, He DZZ, Sziklai I, Metha S, Evans BN (1997) Acetylcholine, outer hair cell electromotility, and the cochlear amplifier. J Neurosci 17:2212-2226.

Dulon D, Zajic G, Schacht J (1990) Increasing intracellular free calcium induces circumferential contractions in isolated cochlear outer hair cells. J Neurosci 10:1388-1397.

Forge A (1991) Structural features of the lateral walls in mammalian cochlear outer hair cells. Cell Tissue Res 265:473-483.

Frolenkov GI, Kalinec F, Tavartkiladze GA, Kachar B (1997) Cochlear outer hair cell bending in an external electric field. Biophys $\mathbf{J}$ 73:1665-1672.

Frolenkov GI, Atzori M, Kalinec F, Mammano F, Kachar B (1998) The membrane-based mechanism of cell motility in cochlear outer hair cells. Mol Biol Cell 9:1961-1968.

Frolenkov GI, Mammano F, Belyantseva IA, Coling D, Kachar B (2000) Two distinct $\mathrm{Ca}^{2+}$-dependent signaling pathways regulate the motor output of cochlear outer hair cells. J Neurosci 20:5940-5948.

Geleoc GSG, Casalotti SO, Forge A, Ashmore JF (1999) A sugar transporter as a candidate for the outer hair cell motor. Nat Neurosci 2:713-719.

Gulley RL, Reese TS (1977) Regional specialization of the hair cell plasmalemma in the organ of Corti. Anat Rec 189:109-123.

He DZ, Evans BN, Dallos P (1994) First appearance and development of electromotility in neonatal gerbil outer hair cells. Hear Res 78:77-90.

Holley MC, Kalinec F, Kachar B (1992) Structure of the cortical cytoskeleton in mammalian outer hair cells. J Cell Sci 102:569-580.

Huang G, Santos-Sacchi J (1993) Mapping the distribution of the outer hair cell motility voltage sensor by electrical amputation. Biophys $\mathrm{J}$ 65:2228-2236.

Kachar B, Brownell WE, Altschuler R, Fex J (1986) Electrokinetic shape changes of cochlear outer hair cells. Nature 322:365-368.

Kalinec F, Kachar B (1993) Inhibition of outer hair cell electromotility by sulfhydryl specific reagents. Neurosci Lett 157:231-234.

Kalinec F, Holley MC, Iwasa KH, Lim DJ, Kachar B (1992) Membranebased force generation mechanism in auditory sensory cells. Proc Natl Acad Sci USA 89:8671-8675.

Nakazawa K, Spicer SS, Schulte BA (1995) Postnatal expression of the facilitated glucose transporter, GLUT-5, in gerbil outer hair cells. Hear Res 82:93-99.

Newsholme EA, Leech AR (1983) Biochemistry for the medical sciences, pp 325-330. New York: Wiley.

Oliver D, Fakler B (1999) Expression density and functional characteristics of the outer hair cell motor protein are regulated during postnatal development in rat. J Physiol (Lond) 519:791-800.

Pujol R, Carlier E, Lenoir M (1980) Ontogenetic approach to inner and outer hair cell function. Hear Res 2:423-430.

Rubel EW (1984) Ontogeny of auditory system function. Annu Rev Physiol 46:213-229.

Rueda J, Prieto JJ, Merchan JA (1990) Development of glycogen content in normal and hypothyroid organ of Corti. Adv Otorhinolaryngol 45:140-142.

Russ JC (1999) The image processing handbook, pp 194-199. Boca Raton, FL: CRC.

Santos-Sacchi J (1991) Reversible inhibition of voltage-dependent outer hair cell motility and capacitance. J Neurosci 11:3096-3110.

Schulte BA (1993) Immunohistochemical localization of intracellular $\mathrm{Ca}$-ATPase in outer hair cells, neurons and fibrocytes in the adult and developing inner ear. Hear Res 65:262-273.

Uziel A, Romand R, Marot M (1981) Development of cochlear potentials in rats. Audiology 20:89-100.

Zheng J, Shen W, He DZZ, Long KB, Madison LD, Dallos P (2000) Prestin is the motor protein of cochlear outer hair cells. Nature 405: $149-155$. 